\title{
Objective Dysphonia Quantification in Vocal Fold Paralysis: Comparing Nonlinear with Classical Measures
}

\author{
Max A. Little*, Declan A. E. Costello ${ }^{\star}$, and Meredydd L. Harries ${ }^{\ddagger}$ \\ * Systems Analysis, Modeling and Prediction Group, University of Oxford, Oxford, United \\ Kingdom \\ tOxford Deanery, Oxford, United Kingdom \\ ‡ Royal Sussex County Hospital, Brighton, United Kingdom.
}

\section{Summary}

Clinical acoustic voice-recording analysis is usually performed using classical perturbation measures, including jitter, shimmer, and noise-to-harmonic ratios (NHRs). However, restrictive mathematical limitations of these measures prevent analysis for severely dysphonic voices. Previous studies of alternative nonlinear random measures addressed wide varieties of vocal pathologies. Here, we analyze a single vocal pathology cohort, testing the performance of these alternative measures alongside classical measures. We present voice analysis pre- and postoperatively in 17 patients with unilateral vocal fold paralysis (UVFP). The patients underwent standard medialization thyroplasty surgery, and the voices were analyzed using jitter, shimmer, NHR, nonlinear recurrence period density entropy (RPDE), detrended fluctuation analysis (DFA), and correlation dimension. In addition, we similarly analyzed 11 healthy controls. Systematizing the preanalysis editing of the recordings, we found that the novel measures were more stable and, hence, reliable than the classical measures on healthy controls. RPDE and jitter are sensitive to improvements pre- to postoperation. Shimmer, NHR, and DFA showed no significant change $(P>$ $0.05)$. All measures detect statistically significant and clinically important differences between controls and patients, both treated and untreated $(P<0.001$, area under curve [AUC] $>0.7)$. Preto postoperation grade, roughness, breathiness, asthenia, and strain (GRBAS) ratings show statistically significant and clinically important improvement in overall dysphonia grade $(\mathrm{G})$ (AUC $=0.946, P<0.001$ ).

Recalculating AUCs from other study data, we compare these results in terms of clinical importance. We conclude that, when preanalysis editing is systematized, nonlinear random measures may be useful for monitoring UVFP-treatment effectiveness, and there may be applications to other forms of dysphonia.

\section{Keywords}

Nonlinear analysis; Random analysis; Voice analysis; Vocal fold paralysis; Jitter; Shimmer; Noise-to-harmonic ratio

\footnotetext{
(C) 2009 The Voice Foundation
}

Address correspondence and reprint requests to Declan A.E. Costello, Department of Otolaryngology, John Radcliffe Hospital, Headley Way, Oxford OX3 9DU, United Kingdom. declancostello@ doctors.org.uk. 


\section{INTRODUCTION}

Unilateral vocal fold paralysis (UVFP) is a voice disorder that typically results in breathy, hoarse, or rough voice and the general inability to produce clear phonation. The weakness of one side of the larynx prevents proper adduction of both folds necessary to sustain vibration. The condition is often disabling and distressing and can affect the quality of life. Surgical intervention in the form of medialization is, however, often effective at restoring good vocal function.

Before and after any intervention, assessment of patients in the voice clinic should cover several areas: the vocal tract should be examined videostroboscopically; the patient should self-rate his or her voice (using, eg, the voice handicap index) $;^{1}$ and the clinician should make an assessment of the patient's voice, using a rating tool, such as the (perceptual) grade, roughness, breathiness, asthenia, and strain (GRBAS) scale. ${ }^{2}$ A summary of the recommended tools for assessment is presented in the European Laryngological Society guidelines. ${ }^{3}$ Although the use of the GRBAS scale is recommended, objective measures of acoustic voice recordings made in the clinic are also important, not only to quantify the degree of dysphonia, but to monitor the patient's response to treatment. This article addresses the broad question of the suitability of measures for characterizing UVFP objectively.

To obtain objective measures, a digital acoustic recording of the voice is analyzed using mathematical algorithms, resulting in a set of numbers, each number characterizing a specific aspect of the voice. ${ }^{4}$ Such measures are valuable because, under controlled clinical conditions and all other things being equal, an objective measure depends only on the voice recording and the details of the algorithm, and bypasses the many subjective aspects of perception and interpretation of the individual clinician inherent to provide a GRBAS score. ${ }^{5}$ Hence, objective measures can provide an additional level of consistency in the quantification and monitoring of dysphonia.

There are a large number of algorithms for the objective analysis of voice, but perhaps, the most dominant in clinical practice are the classical jitter, shimmer, and noise-to-harmonic ratio (NHR) (perturbation) measures, ${ }^{4}, 6$ and their many variants, based on mathematical signal-analysis techniques. Signal analysis itself being of utility across many scientific disciplines, there are many broad mathematical signal-analysis frameworks. Two frameworks have traditionally formed the basis of the objective voice measures mentioned earlier: the classical concepts of waveform-based cycle analysis ${ }^{4},{ }^{7}$ and linear digital signal processing. ${ }^{8}$ However, all frameworks make certain mathematical assumptions about the signal that may not hold in reality, ${ }^{7}$ so that objective algorithms will have practical limitations stemming partly from the specific assumptions of their underlying frameworks.

From a biophysical standpoint, the celebrated source-filter model of voice production isolates the vocal folds and the vocal tract as separate components, with the laryngeal source driving the tract (modeled as a linear system) into resonance at specific formant frequencies. ${ }^{9}$ The natural pairing of the linear source-filter model with the linear signal-processing framework is of great utility in a wide variety of applications, ${ }^{9}-11$ including objective clinical voice analysis. ${ }^{4},{ }^{7}$ However, at least three decades ago, it was realized that nonlinearity in biophysical models was required to account for the observed motion of the vocal folds, ${ }^{12}{ }^{15}$ and that the idealized separation of folds and tract into separate components misrepresents observed nonlinear feedback interactions between the two. ${ }^{10}, 13$ Subsequent biophysical modeling ${ }^{16} \_20$ and empirical voice signal-analysis studies ${ }^{21} \_26$ discovered a wealth of characteristically nonlinear phenomena produced by the vocal system. 
Furthermore, the voice involves turbulent airflow in the vocal organs, turbulence that is critical to the production of consonants and aeroacoustic noise (breath noise), which is a pervasive feature of voice production. ${ }^{13},{ }^{27}{ }^{29}$ Thus, there is compelling evidence for nonlinearity and randomness as inherent features of voice production, both in models and signals.

By definition, nonlinear phenomena are not naturally suited to linear signal-processing analysis. ${ }^{13}$ Nonlinear waveforms are also characteristically nonrepetitive and complex. ${ }^{30}$ Thus, neither are they suited to cycle analysis, which assumes that the signal is nearly periodic (showing a nearly repetitive waveform ${ }^{7}$ ). This mismatch between mathematical signal-analysis framework and signal characteristics is of particular relevance to clinical practice, because it is precisely the mild-to-severe dysphonic pathological voices, such as in UVFP, which show highly nonlinear and random phenomena ${ }^{13},{ }^{25}$ - healthy voice signals are often nearly periodic and hence more suited to perturbation measures based on cycle analysis.

These limitations of cycle- and linear-analysis frameworks for pathological voices have motivated the framework of nonlinear time series analysis ${ }^{31}$ for objective voice measurement, ${ }^{13},{ }^{14},{ }^{17},{ }^{25}, 32 \_37$ more recently extended to encompass random motion as well. $13,14,38$ 40 This new framework of nonlinear, random systems analysis is well suited to analyze the full range of nonlinear and noisy phenomena observed in pathological voices, $13,{ }^{14}$ where the signals range from strictly periodic (repetitive) to highly aperiodic (nonperiodic) and random. This is important in practice because when the assumptions of cycle or linear analysis no longer hold, as will be the case for highly breathy, rough, or otherwise dysphonic voices, an objective measure based on this framework can fail to return a number, or, which is often worse, return a spurious number which, rather than reflecting the severity of the dysphonia, responds to some unanticipated interaction between the specifics of the analysis algorithm and the peculiarities of the signal. ${ }^{13},{ }^{41}$

Novel objective measures based on the nonlinear random framework, such as recurrence period density entropy (RPDE) and detrended fluctuation analysis (DFA), have recently been devised, whose output is rigorously characterized for all signals; from the strictly periodic, through nearly periodic, to highly aperiodic and purely random signals, on a fixed numerical scale with finite lower and upper limits. ${ }^{14}$ Thus, in theory, such measures are valuable to clinical practice because of their wide applicability to all voice signals, not just those that are nearly periodic and hence amenable to perturbation analysis.

In nontechnical terms, DFA characterizes the changing detail of aeroacoustic breath noise in the voice. It is, therefore, sensitive to similar features in the voice as NHR. In contrast, RPDE rigorously quantifies any ambiguity in fundamental pitch that might exist, and this is useful because an increasing level of ambiguity is often indicative of vocal dysfunction. For nearly periodic voices, RPDE and jitter measure similar properties of the signal. Correlation dimension can be thought of as a measure of the overall complexity of a voice signalperiodic signals display a single, simple oscillating pattern, and will, therefore, have low dimension. As the voice becomes more dysphonic and hence aperiodic, the patterns of oscillation become harder to predict, and the dimension increases.

Given the potential of such novel measures, previous studies ${ }^{14},{ }^{42}$ have examined their performance on a wide variety of vocal pathologies alongside classical perturbation measures. The aim has been to test the relative effectiveness of these novel measures for discriminating pathological voices from healthy controls compared with classical measures. This has demonstrated that the novel measures are at least as, and often more, effective, than classical measures at discriminating healthy from general dysphonic voices. ${ }^{14}, 40$ 
However, an analysis of the relative reliability of such measures is missing in the literature: for healthy voices, by how much do they vary compared with classical measures? That is, how relatively consistent are they when applied to controlled signals from healthy voices? Next is the question of relative sensitivity: can these novel measures detect changes in dysphonia, pre- and postoperatively for UVFP, and in this respect, are they more or less sensitive than classical measures? Finally, do these measures conform in any way to perceptual rating scales? Obtaining answers to these questions is a necessary precondition for clinical usefulness in UVFP.

Previous studies have demonstrated a statistically significant change in nonlinear measures applied to healthy controls and untreated UVFP patients, ${ }^{33}$ demonstrating that nonlinear measures hold some promise. Furthermore, recent studies suggest that nonlinear measures correlate well with perceptual evaluation by experienced listeners ${ }^{37}$ for a broad range of voice disorders. Nonetheless, the main questions mentioned earlier remain unanswered.

Hence, we compare pre- and postoperative patients with healthy controls using the same nonlinear measure, two novel nonlinear random measures, and the same classical perturbation measures as in Zhang et al. ${ }^{33}$ We test the relative reliability of these measures on the healthy controls. We pay careful attention to control the confounding factors, such as inhomogeneity of patient selection, pathological process, and treatment regime, focusing on an accepted, standardized surgical intervention applied uniformly across the whole group. We also avoid the potentially confounding effect of selective digital audio editing that can occur when preparing the recordings for objective analysis. Finally, blinded GRBAS ratings from three different clinical raters were obtained pre- and postoperatively to compare against the objective results.

Our study, therefore, has several aims:

1. To assess the reproducibility of classical (jitter, shimmer) and nonlinear (RPDE $[\mathrm{H}]$, correlation dimension, and DFA $[a])$ methods in analyzing the voices of normal subjects;

2. To compare classical and nonlinear methods in analyzing the voices of UVFP subjects, pre- and postoperatively;

3. To establish whether any changes in the classical and nonlinear analyses are matched by changes in perceptual ratings (GRBAS);

4. To compare our results with the existing literature.

To our knowledge, this is the first direct assessment of the relative performance of a range of novel nonlinear techniques alongside classical perturbation measures, comparing pre- and postoperative UVFP voices.

\section{METHODS}

\section{Demographics and recording protocol}

We recorded the voices of 17 adult patients (nine male and eight female) with UVFP. Acoustic recordings were made using the Laryngograph system (www.laryngograph.com, London, UK), under standardized conditions, in the same quiet room. A microphone (Knowles EK-3132 (Itasca, IL), omnidirectional electret condenser type) was placed 16.5 $\mathrm{cm}$ from the patient's mouth and recordings were taken $(22.05 \mathrm{kHz}, 16 \mathrm{bits})$. We also recorded 11 healthy male and female controls under similar quiet conditions.

All subjects were recorded sustaining the vowel /a/ at a loudness and pitch that was comfortable to them and was at approximately conversational level. We obtained 166 
recordings of separate vowel phonations, an average of nearly six phonations per subject. All UVFP patients were recorded reading the standard running speech test "The North Wind the and Sun" for the purposes of GRBAS scoring alone.

\section{Surgical intervention}

Each UVFP patient underwent a standard operative procedure: Isshiki type 1 (medialization) thyroplasty. ${ }^{43}$ A standard sedative (a propofol, remifentanil, and alfentanil infusion) was administered, and the procedure was performed under local anesthesia. A transverse skin incision was made at the level of the cricothyroid membrane and the strap muscles were retracted laterally. The thyroid lamina was exposed. A window was cut (or drilled, in the case of calcified cartilages) and the internal periosteum was elevated. Through the window in the thyroid cartilage, a silastic shim was inserted to medialize the paralyzed vocal fold. Before insertion, the shim was cut to an appropriate size for the individual patient, depending on the size of the glottic gap seen on laryngoscopy. With the shim in situ, the sedation was reduced until the patient was lightly sedated and able to phonate. The patient was then asked to perform some vocal tasks, and if the voice was deemed to be too breathy, a larger shim was cut and inserted. The skin was closed in layers with no drain. All cases were performed by the same surgeon (M.L.H.) and patients were discharged home within 4 hours of surgery.

\section{Postoperative recording}

After a period of recuperation and acclimatization, the UVFP patients' voices sustaining the vowel /a/ and reading the running speech test were re-recorded several months after surgery under the same acoustic conditions.

\section{Preparation of recordings-systematic trimming}

At the onset of a vocal gesture, the voice may take a fraction of a second to stabilize. To circumvent any confounding effect of spurious instability in phonation at the very start and end of phonations, 0.12 seconds of the signal at the start and end of phonations was removed from every recording. Figure 1 illustrates this "trimming" process. This 0.12 -second trimming was applied systematically across all phonations from both healthy and UVFP subjects.

\section{Grade, roughness, breathiness, asthenia, and strain rating}

Three experienced speech and language therapists (blinded as to whether the patients were pre- or postoperative) independently rated each of the pre- and postoperative voice recordings (both sustained vowels and running speech tests- "The North Wind and the Sun" passage) using the GRBAS scale. The overall grade $(\mathrm{G})$, roughness $(\mathrm{R})$, breathiness (B), asthenia (A), and strain (S) were each evaluated and assigned a value of $0,1,2$, or 3 (where 0 corresponds to "none" and 3 to "severe").

\section{Objective measures}

Sustained phonations from all subjects were analyzed using objective measures, pre- and postoperatively for UVFP patients. Six measures: (1) jitter (ABS), (2) shimmer (dB), (3) NHR, (4) RPDE, (5) DFA, and (6) correlation dimension were calculated for each sustained vowel recording. The first three perturbation measures were calculated using the Praat software system. ${ }^{44}$

Perturbation methods: jitter, shimmer, and noise-to-harmonic ratio-Praat s perturbation measures are based on estimating cycle lengths using the waveform-matching algorithm, searching for the best match between successive cycles, occurring at a cross- 
correlation maximum. Hence, the measures are based on a combination of the two frameworks of cycle analysis (assuming that the signal is composed of successive similar cycles), and classical linear signal processing (for estimating the extent of the match between putative cycles). The jitter (ABS) algorithm is the average absolute difference between consecutive cycle lengths, in seconds. For reference purposes, this coincides with the Kay Pentax Multi-Dimensional Voice Program "Jita" parameter, ${ }^{45}$ where 83.200 microseconds is given as the lower limit for pathological voice. The shimmer (dB) parameter is 20 times the average absolute (base 10) logarithm of the difference between the amplitudes of successive cycles, in decibels $(\mathrm{dB})$. This coincides with the MDVP parameter "ShdB," where $0.350 \mathrm{~dB}$ is suggested as the lower limit for pathology. The NHR algorithm is calculated as the average inverse harmonics-to-noise ratio (HNR) over each cycle. The (windowed) autocorrelation is calculated and the time lag at which the autocorrelation achieves a global maximum is found. The HNR for each cycle is 10 times the logarithm of the ratio of the maximum autocorrelation to one minus this maximum autocorrelation value. ${ }^{46}$ For further details, see Boersma. ${ }^{46}$

Nonlinear analysis methods: recurrence period density entropy $(\mathrm{H})$, detrended fluctuation analysis ( $\alpha$ ), and correlation dimension-The RPDE and correlation dimension measures are based on the framework of nonlinear time-series analysis, which assumes that the signal is generated by a model of the physical processes of voice production itself (expressed as a set of nonlinear differential equations optionally driven by a random input). They admit a larger class of signals than the cycle or linear frameworks, but include signals that conform to both of these frameworks as special cases. An attempt is made to infer properties about a nonlinear (and optionally random) model for the physical processes that generated the voice signal by constructing a time-delay embedding (ie, a set of vectors constructed from time-lagged copies of the signal). Four time-lagged copies were used at a time lag of 0.002 seconds; these embedding parameters were estimated using a brute-force search procedure to satisfy known special cases. ${ }^{14}$

From the lagged vectors, RPDE estimates the relative uncertainty in the expected recurrence periods of the embedded signal, that is, the time intervals between successive close returns to the same point in the space spanned by the lagged vectors. It can be shown that this generalizes the notion of cycle length for cyclic signals, and period for exactly periodic signals. ${ }^{14}$ Thus, both cyclic and periodic signals are special cases of recurrent signals. RPDE then constructs a distribution of all recurrence times. Exactly periodic signals have only one recurrence period; hence, the relative entropy (uncertainty with respect to uniformly random recurrence times, denoted $H_{\text {norm }}$ ) of the distribution will be zero, indicating no uncertainty about the recurrence period (cycle length/exact period) of the signal. Nearly periodic signals will show some spread of recurrence periods - the uncertainty will be slightly larger.

Aperiodic signals will show a typically large spread of recurrence periods, and hence, have a still larger $H_{\text {norm. }}$. At the most extreme level, a completely random signal shows recurrence periods of all lengths, with larger periods being more rare than smaller periods, and $H_{\text {norm }}$ being at most unity.

Of fundamental interest in the nonlinear time-series-analysis framework are chaotic signals that are often characteristically aperiodic. ${ }^{30}$ In the lagged vector space, many of these chaotic signals have fractal dimension (ie, they occupy less space than a 3D object, but more space than a flat, 2D object). Evidence points to many examples of aperiodic voice signals that appear chaotic. ${ }^{17}{ }^{19},{ }^{24},{ }^{25}$ Thus, measuring the dimension of the signal in the lagged space might characterize voice disorders usefully, assuming that healthy voices will be very close to periodic voices, and hence, have low dimension, and that dysphonic signals will have a much higher dimension. The correlation dimension measure is one approach to 
estimate this lagged space dimension $D$; here, we use the correlation dimension algorithm proposed by Judd. ${ }^{47}$

Turbulence is typically characterized by persistent random fluctuations on all time and spatial scales - a commonly held theory proposes that the logarithm of the magnitude of these fluctuations scales linearly with the logarithm of the temporal scale. ${ }^{48}$ This is a defining property of random fractals signals, and many dysphonic voice signals show this kind of behavior on small time scales. Measuring the acoustic manifestation of this turbulence contributes to the detection of dysphonia. ${ }^{13},{ }^{14},{ }^{40}$ DFA attempts to measure this scaling factor $a$ in the fine scale detail of the noise in the voice signal. Here, the scale factor is normalized by a nonlinear transformation to lie in the range of zero to unity (the normalized scale factor is denoted as $a_{\text {norm }}$ ).

\section{Relative reliability of objective measures}

Addressing the question of comparative reliability of the objective measures, the (fractional) variability of each measure applied to the healthy sustained phonations was calculated. The variability was estimated nonparametrically as the interquartile range (IQR: the difference between the 75th and 25th percentile) divided by the median of all healthy phonations. To test the effect of altering the systematic trimming during the preparation of the recordings described earlier, the variability for each measure was calculated as the trimming length was varied from 0.04 to 0.28 seconds in 0.04 -second steps.

\section{RESULTS}

Table 1 shows the reliability analysis results, assessed using the fractional variability of each objective measure applied to all the healthy phonations. The DFA measure shows the smallest variability, and the NHR the largest variability across all healthy subjects. The median variability across all measures is somewhat sensitive to the systematic trimming time used in the preparation of the recordings.

Next, Figure 2 shows the perceptual GRBAS scoring results for the UVFP patients. The total, breathiness, asthenia, and grade GRBAS scores show clear separation between preand postoperation. The strain and roughness subcategories do not show clear separation.

Figure 3 gives a graphical representation of the objective measures applied to both healthy and UVFP subjects (both pre- and postoperatively). It can be seen that the RPDE measure shows visibly clear changes from untreated (solid black line) to treated (broken black line) recordings, mirroring the GRBAS results. For jitter and NHR, in contrast, the shift before and after treatment is not so clear. The DFA and NHR measures show no clear, visible change between untreated and treated patients. On comparing untreated and healthy voices, a visible shift in the curve to the left can be seen with all measures except NHR, although RPDE and dimension show the largest changes.

Table 2 lists summary statistics for the measures. Both median and mean of all measures show differences pre- and post-operation, and Table 3 details the corresponding numerical results of the statistical analysis. Because of the heavy non-normality of the data, statistical significance and effect sizes are assessed nonparametrically, significance using the rank-sum test, and effect size using the receiver operating characteristic area under curve (ROC AUC), which is a measure of the overlap between categories, in that an AUC value of unity indicates that it is possible to have perfect separability between categories, and an AUC of 0.5 indicates that we can do no better than uniformly random classification. ${ }^{49}$ 
The aforementioned graphical findings are confirmed in that GRBAS total $(P<0.001)$, breathiness $(P<0.001)$, asthenia $(P<0.001)$, grade $(P<0.001)$, RPDE $(P=0.03)$, and jitter $(P=0.03)$ all show a statistically significant $=$ change $(95 \%$ significance level $)$ pre- and postoperatively (treated vs untreated columns in Table 2). The largest effect size (AUC) corresponds to the most clinically important change, and this is achieved by the GRBAS grade score $(A U C=0.946)$. Of the objective measures, only RPDE and jitter show a significant change and are of equal importance $(\mathrm{AUC}=0.634)$. All objective measures show a significant and clinically important difference when detecting normal versus UVFP, either treated or untreated.

\section{DISCUSSION}

The first question posed in the introduction asked about the relative reliability of the objective measures applied to healthy controls. The results shown in Table 1 are interesting because they demonstrate that the novel nonlinear (random) measures show considerably less variability as a fraction of their median values than the classical perturbation measures tested here. In fact, the least variable of these novel measures (DFA) shows almost one-sixth the variability of NHR. This may not be of particular consequence here, because DFA could not detect the pre- to postoperative improvement, but perhaps, more importantly, the only perturbation measure significantly able to detect a pre- to postoperative change in UVFP (jitter) is nearly three times as variable as the correlation dimension measure.

The next question posed is one of relative sensitivity to the voice changes evidenced by GRBAS scoring in these UVFP patients postintervention. Figure 3 and Table 3 clearly demonstrate that although it is not possible to achieve the same level of clear discrimination as perceptual scoring, of all the objective measures, the novel RPDE and jitter measures are the most indicative.

The final question asks how the objective measures relate to the perceptual scores. Here, we can see that the RPDE and jitter measures best reflect the large change detected pre- and postoperatively, discriminating between categories with about two-thirds the separability of the GRBAS grade score.

We now investigate how these results sit with other studies. Several general comments about comparisons across studies must be made first. Our first observation, highlighted by our reliability results, is that perturbation methods are subject to inherently large variability, even for controlled, healthy voices. For example, a recent study showed poor test-retest reliability (ie, the consistency of the measure without intervention). ${ }^{41}$ Thus, several repetitions of the sustained phonation are required to minimize sampling variability even in the absence of pathology. Here we take, on average, six phonations per subject, and include all phonations in the overall statistical analysis. Other studies do not do this, ${ }^{50}$ or take the mean measure over three phonations, ${ }^{51}$ or take a single phonation with the lowest jitter measure. ${ }^{52}$

Similarly, although "jitter" and "shimmer" appear to name one algorithm, this disguises a plethora of different algorithms, across many different software vendors. ${ }^{41}$ Seemingly trivial differences in cycle-length measurement can give very different measures. ${ }^{7}$ Many studies give no algorithm details.

Furthermore, untreated UVFP voices can be so dysphonic that the cycle and/or linear signalprocessing mathematical framework becomes inapplicable, and no perturbation analysis is possible. ${ }^{6},{ }^{33},{ }^{41}$ In this study, for example, one of the preoperative voice recordings was very severely dysphonic, and shimmer measurements were not possible at all. Thus, it is difficult to get statistically powerful results, particularly for the preoperative category. Often, this 
forces hand editing of segments of the voice recording (described in, eg, Uloza et $\mathrm{al}^{51}$ ) so that perturbation analysis becomes possible. However, this practice is inherently subjective, and thus, may introduce subtle and unintended selection biases. Hand-editing methodology also differs considerably, some studies analyzing a "middle" section and discarding undefined starts and ends, ${ }^{51}, 52$ or selecting "the most stable 0.5 seconds," 52 without further methodological clarification. Our reliability results suggest that a systematic, algorithmic approach to editing the voice signal for objective analysis is necessary, because the variability of measures depends on which parts of the recording are included for analysis.

Finally, comparing studies on the results of statistical significance tests alone is of limited usefulness in practice, because a small but uninteresting effect can reach a high level of significance with large sample size; conversely, a clinically important effect can be statistically insignificant solely because of a small number of subjects. ${ }^{53}$ We survey some representative studies and reanalyze reported data to make direct comparisons with our results by calculating significances and AUC values from reported category means and standard deviations (SDs), ${ }^{49}$ assuming their data to be normal.

Previous work using nonlinear measures has tended to focus on a broad range of pathologies and few studies have examined changes after therapy. However, Zhang et $\mathrm{al}^{34}$ investigated a cohort of patients with vocal fold polyps. In that study, jitter, shimmer, and two nonlinear measures (correlation dimension and second-order entropy) were undertaken before and after surgery. The nonlinear measures showed significant decrease after surgery. Shimmer showed no significant change after surgery, but jitter did. These aspects of the study agree with our results. Unfortunately, direct comparisons of effect size are not possible because full summary statistics for the data are not disclosed.

A further study ${ }^{33}$ tested the performance of correlation dimension against jitter and shimmer on voices of patients with UVFP and healthy controls. Jitter and shimmer were calculated only for that subset of voices that were nearly periodic, and correlation dimension was calculated for all the voices in the study. All three measures showed a statistically significant difference between healthy controls and UVFP, with shimmer showing the largest AUC, and jitter and correlation dimension showing a lower (but similar) effect. Our study confirms these findings (see last row in Table 1) from the view of significance alone, but we find much larger AUC values. We note that in that study, jitter and shimmer could not be applied to the very dysphonic voices.

Across studies, jitter is found to have a significant change pre- to postsurgery ${ }^{50}{ }^{52}, 54$ ranging in effect size from AUC $=0.634$ to 0.974 , a variability that is consistent with Zhang and Jiang. ${ }^{42}$ Our jitter effect is on the lower side. In contrast, studies disagree about the significance of shimmer, and we find no significant effect. All studies agree that NHR shows no statistical change after thyroplasty.

Most of these studies assume that the normal distribution is a good model for the data. The high variability and low consistency of perturbation measures discussed earlier often generate large outliers. Coupled with the fact that our data are highly non-normal, this raises doubts about the validity of such nonrobust statistical methods. No normality-test results or plots of the data are provided in these studies. As an illustration of the sort of variability that can occur, we calculated normal Student's $t$ test significances and compared them with our rank-sum results: normal assumptions then admit both dimension and NHR as being significant, even though their AUCs are too small to be interesting.

Previous studies have shown that both RPDE and DFA are useful in separating healthy voices from dysphonic voices of many causes. ${ }^{13},{ }^{14}$ However, DFA did not show a significant change pre- and postoperatively in UVFP, although, as with all the other 
measures, it did detect a change between healthy and dysphonic voices. This contributes an additional layer of detail to other studies which found broad differences in DFA between healthy and pathological voices.

One of the strengths of the present study is the homogeneity of the patients: all had a defined pathology that was managed with a uniform and repeatable operation. This study, therefore, avoids many of the confounding factors seen in studies of other vocal pathologies. To our knowledge, this is the first direct assessment of the relative performance of a range of novel nonlinear techniques alongside classical perturbation measures, comparing pre- and postoperative UVFP voices.

Nonlinear objective measures have additional benefits for many clinical applications. Most importantly, the nonlinear framework does not rely on near periodicity of the acoustic signal, and is, consequently, applicable to the full range of periodic to highly aperiodic voices typically encountered in clinical practice. Furthermore, measures, such as RPDE and DFA, produce outputs constrained to lie on a fixed 0-1 numerical scale, and this is useful for clinical communication of the severity of dysphonic symptoms.

We now provide some notes on interpretation of these novel nonlinear (random) measures. Classical perturbation methods based on cycle analysis might best be described as morphological methods, that is, they are designed to find and characterize specific kinds of sequences of waveform shapes in a signal (when considered as a graph of acoustic pressure against time). Although these sequences of waveforms are observed in most voice signals, there is no theoretical basis that affords confidence that the vocal system will not produce waveforms falling outside these narrowly defined sequences. Thus, cycle-analysis methods require near periodicity, but many real voice signals are far from near periodic, for wellmotivated physiological reasons. ${ }^{13}$

In contrast, nonlinear (random) methods assume that there is an underlying mechanistic or random process that generated the recording, and attempt to reconstruct and characterize aspects of this underlying process from the recorded signal. In particular, RPDE measures one aspect of the recurrence of the underlying physical state of the vocal system, that is, qualitatively, the extent to which it repeats the same sequence of configurations and momentum, without specifying in advance the signal morphology of these constituent sequences. One typical example is subharmonic vibration, which can naturally develop even in normal phonation. This has an ambiguous interpretation within the linear framework because, often, the subharmonic has nearly the same amplitude in the power spectrum as the fundamental pitch. RPDE will typically detect both fundamental and subharmonic, but RPDE will show an increased uncertainty value $H_{\text {norm }}$ relative to phonation without subharmonics. Thus, in this situation, RPDE rigorously quantifies the genuine ambiguity in fundamental pitch, and this ambiguity is often indicative of vocal dysfunction.

DFA characterizes the changing detail of aeroacoustic breath noise in the voice. Although there are a very large number of classical noise measures, these are usually based on spectral analysis using linear signal processing. Aperiodic dysphonia typical of chaotic vibration is inseparable from aspiration noise in spectral analysis, ${ }^{13}$ even though the chaotic signal can be entirely smooth in the time domain. DFA is sensitive to the temporal details of the signal alone, whether or not the slow vibrational motion in the vocal system is aperiodic, nearly periodic, or strictly periodic. ${ }^{13}$ Excessive aeroacoustic noise is a common feature of many dysphonias, the underlying physical origin being the turbulent airflow in the larynx and vocal tract, ${ }^{27}{ }^{29}$ often enhanced by incomplete vocal fold closure and aphonia in severe UVFP cases. ${ }^{41}$ 
All mathematical frameworks have limitations, including the nonlinear (random)

framework. Here, we remark that RPDE and correlation dimension will not be applicable to completely silent signals, or more generally, to those that show less than one full repetition. In addition, correlation dimension requires sufficiently noise-free recordings. Similarly, DFA requires the signal to have enough bandwidth to detect any scaling properties in the fine-scale detail. Nonetheless, these limitations are far less restrictive than those that must apply for reliable classical perturbation analysis.

\section{CONCLUSION}

In this study, we compared classical objective acoustic measures for UVFP dysphonia based around the cycle and linear signal-processing mathematical frameworks, against novel measures based on the framework of nonlinear (random) time-series analysis. We tested the reliability of all these measures on healthy controls, and found that the nonlinear methods were more stable and reproducible than the classical measures. We then quantified the performance of these measures in detecting improvements in dysphonia after surgical treatment for UVFP in a cohort of patients that showed perceptually rated improvements according to standardized expert clinical judgment. Our main finding is that, when potential systematic biases because of hand editing of acoustic recordings were circumvented, only one of the nonlinear measures and one of the perturbation measures were able to provide a statistically significant detection of this change, although this was dependent on the statistical assumptions. This measure has, however, comparable performance to the only classical perturbation measure shown to be able to detect the change consistently. The main contribution of this study is the provision of further evidence that nonlinear objective measures are able to perform at least as well as their classical counterparts, and that they change consistently with perceptual voice analysis scales. ${ }^{37}$

We believe these results motivate research developing further applications for the nonlinear analysis and additional comparisons with classical measures. It is possible that, with widening experience of these newer measures, they will be available alongside classical measures in commercially available software packages, where they can provide more sophisticated, objective tracking and monitoring of dysphonia in the treatment of voice disorders. We conclude with the recommendation that nonlinear random acoustic objective measures should play an increasing role in the assessment protocol in the voice clinic.

\section{Acknowledgments}

We are very grateful to Carol Harris, Caroline Fraser, and Nikki Wright for their assistance with the project.

\section{REFERENCES}

1. Jacobson BH, et al. The Voice Handicap Index (VHI): development and validation. Am J Speech Lang Pathol. 1997; 6:66-70.

2. Hirano, M. Clinical Examination of Voice. Disorders of human communication. Springer-Verlag; Wien; New York: 1981. p. xi

3. Dejonckere PH, et al. A basic protocol for functional assessment of voice pathology, especially for investigating the efficacy of (phonosurgical) treatments and evaluating new assessment techniques. Guideline elaborated by the Committee on Phoniatrics of the European Laryngological Society (ELS). Eur Arch Otorhinolaryngol. 2001; 258:77-82. [PubMed: 11307610]

4. Baken, RJ.; Orlikoff, RF. Clinical Measurement of Speech and Voice. 2nd ed. Singular Thomson Learning; San Diego: 2000. p. xii

5. Hirano M, et al. Acoustic analysis of pathological voice. Some results of clinical application. Acta Otolaryngol. 1988; 105:432-438. [PubMed: 3400446] 
6. Titze, IR. Workshop on acoustic voice analysis: summary statement. 1995.

7. Roark RM. Frequency and voice: perspectives in the time domain. J Voice. 2006; 20:325-354. [PubMed: 16626935]

8. Proakis, JG.; Manolakis, DG. Digital Signal Processing: Principles, Algorithms, and Applications. 3rd ed. Prentice Hall; Upper Saddle River, NJ: 1996.

9. Flanagan, JL. Speech Analysis; Synthesis and Perception. 2nd ed. Springer-Verlag; Berlin, New York: 1972. p. x

10. Quatieri, TF. Prentice-Hall Signal Processing Series. Prentice Hall; Upper Saddle River, NJ: 2002. Discrete-Time Speech Signal Processing: Principles and Practice; p. 781

11. Cox, RV. Speech coding standards. In: Kleijn, W.; Paliwal, K., editors. Speech Coding and Synthesis. Elsevier; Amsterdam; New York: 1995. p. 49-78.

12. Ishizaka K, Flanagan JL. Synthesis of voiced sounds from a two-mass model of the vocal cords. ATT Bell System Tech J. 1972; 51:1233-1268.

13. Little, MA. Biomechanically Informed Nonlinear Speech Signal Processing. University of Oxford; Oxford: 2007.

14. Little MA, et al. Exploiting nonlinear recurrence and fractal scaling properties for voice disorder detection. Biomed Eng Online. 2007; 6:23. [PubMed: 17594480]

15. Titze IR. Human vocal cords-mathematical-model 1. Phonetica. 1973; 28:129-170. [PubMed: 4788091]

16. Herzel $\mathrm{H}$, et al. Nonlinear dynamics of the voice-signal analysis and biomechanical modeling. Chaos. 1995; 5:30-34. [PubMed: 12780151]

17. Jiang JJ, Zhang Y, McGilligan C. Chaos in voice, from modeling to measurement. J Voice. 2006; 20:2-17. [PubMed: 15964740]

18. Jiang JJ, Zhang Y, Stern J. Modeling of chaotic vibrations in symmetric vocal folds. J Acoust Soc Am. 2001; 110:2120-2128. [PubMed: 11681389]

19. Jiang JJ, Zhang Y. Chaotic vibration induced by turbulent noise in a two--mass model of vocal folds. J Acoust Soc Am. 2002; 112:2127-2133. [PubMed: 12430824]

20. Steinecke I, Herzel H. Bifurcations in an asymmetric vocal-fold model. J Acoust Soc Am. 1995; 97:1874-1884. [PubMed: 7699169]

21. Tokuda I, Miyano T, Aihara K. Surrogate analysis for detecting nonlinear dynamics in normal vowels. J Acoust Soc Am. 2001; 110:3207-3217. [PubMed: 11785822]

22. Tokuda I, Tokunaga R, Aihara K. A simple geometrical structure underlying speech signals of the \{Japanese\} vowel a. Int J Bifurcation Chaos. 1996; 6:149-160.

23. Little MA, et al. Testing the assumptions of linear prediction analysis in normal vowels. $\mathrm{J}$ Acoust Soc Am. 2006; 119:549-558. [PubMed: 16454308]

24. Titze I, Baken R, Herzel H. Evidence of chaos in vocal fold vibration, in vocal fold physiology: frontiers in basic science. 1993

25. Herzel H, et al. Analysis of vocal disorders with methods from nonlinear dynamics. J Speech Hear Res. 1994; 37:1008-1019. [PubMed: 7823547]

26. Berry DA, et al. Interpretation of biomechanical simulations of normal and chaotic vocal fold oscillations with empirical eigenfunctions. J Acoust Soc of Am. 1994; 95:3595-3604. [PubMed: 8046149]

27. Krane MH. Aeroacoustic production of low-frequency unvoiced speech sounds. J Acoust Soc Am. 2005; 118:410-427. [PubMed: 16119362]

28. Sinder, DJ. Synthesis of Unvoiced Speech Sounds Using an Aeroacoustic Source Model. Rutgers University; New Jersey, USA: 1999.

29. Zhao W, et al. Computational aeroacoustics of phonation, part I: computational methods and sound generation mechanisms. J Acoust Soc Am. 2002; 112(5 Pt 1):2134-2146. [PubMed: 12430825]

30. Sprott, JC. Chaos and Time-Series Analysis. Oxford University Press; Oxford; New York: 2003. p. xxp. 507

31. Kantz, H.; Schreiber, T. Nonlinear Time Series Analysis. 2nd ed. Cambridge University Press; Cambridge; New York: 2004. p. xvi 
32. Zhang Y, Jiang JJ. Nonlinear dynamic analysis in signal typing of pathological human voices. Electron Lett. 2003; 39:1021-1023.

33. Zhang Y, et al. Perturbation and nonlinear dynamic analyses of voices from patients with unilateral laryngeal paralysis. J Voice. 2005; 19:519-528. [PubMed: 16301098]

34. Zhang Y, et al. Nonlinear dynamic analysis of voices before and after surgical excision of vocal polyps. J Acoust Soc Am. 2004; 115(5 Pt 1):2270-2277. [PubMed: 15139638]

35. Behrman A, Baken RJ. Correlation dimension of electroglottographic data from healthy and pathologic subjects. J Acoust Soc Am. 1997; 102:2371-2379. [PubMed: 9348696]

36. Giovanni A, Ouaknine M, Triglia JL. Determination of largest Lyapunov exponents of vocal signal: application to unilateral laryngeal paralysis. J Voice. 1999; 13:341-354. [PubMed: 10498051]

37. Yu P, et al. Objective voice analysis in dysphonic patients: new data including nonlinear measurements. Folia Phoniatr Logop. 2007; 59:20-30. [PubMed: 17172783]

38. Stark J, et al. Takens embedding theorems for forced and stochastic systems. Nonlinear Anal Theory Methods Appl. 1997; 30:5303-5314.

39. Jackson PJB, Shadle CH. Pitch-scaled estimation of simultaneous voiced and turbulence-noise components in speech. IEEE Trans Speech Audio Processing. 2001; 9:713-726.

40. Little M, et al. Suitability of dysphonia measurements for telemonitoring of Parkinson's disease. IEEE Trans Biomed Eng. 2008

41. Carding PN, et al. The reliability and sensitivity to change of acoustic measures of voice quality. Clin Otolaryngol Allied Sci. 2004; 29:538-544. [PubMed: 15373870]

42. Zhang Y, Jiang JJ. Acoustic analyses of sustained and running voices from patients with laryngeal pathologies. J Voice. 2008; 22:1-9. [PubMed: 16978835]

43. Isshiki N, et al. Thyroplasty as a new phonosurgical technique. Acta Otolaryngol. 1974; 78:451457. [PubMed: 4451096]

44. Boersma, P.; Weenink, D. Praat: doing phonetics by computer. 2008.

45. KayPENTAX. Kay Elemetrics Disordered Voice Database, Model 4337. Kay Elemetrics; Lincoln Park, NJ: 1996-2005.

46. Boersma, P. Proceedings of the Institute of Phonetic Sciences. University of Amsterdam; 1993. Accurate short-term analysis of the fundamental frequency and the harmonics-to-noise ratio of a sampled sound.

47. Judd K. An improved estimator of dimension and some comments on providing confidence intervals. Phys D Nonlinear Phenomena. 1992; 56:216-228.

48. Falconer, KJ. Fractal Geometry: Mathematical Foundations and Applications. Wiley; Chichester; New York: 1990. p. xxii

49. Marzban C. The ROC curve and the area under it as performance measures. Weather Forecast. 2004; 19:1106-1114.

50. Dursun G, et al. Long-term results of different treatment modalities for glottic insufficiency. Am J Otolaryngol. 2008; 29:7-12. [PubMed: 18061825]

51. Uloza V, Pribuisiene R, Saferis V. Multidimensional assessment of functional outcomes of medialization thyroplasty. Eur Arch Otorhinolaryngol. 2005; 262:616-621. [PubMed: 16133469]

52. Shin JE, et al. Analysis of voice and quantitative measurement of glottal gap after thyroplasty type I in the treatment of unilateral vocal paralysis. J Voice. 2002; 16:136-142. [PubMed: 12002881]

53. Gardner MJ, Altman DG. Confidence-intervals rather than P-values-estimation rather than hypothesis-testing. BMJ. 1986; 292:746-750. [PubMed: 3082422]

54. Lu FL, et al. Longitudinal evaluation of vocal function after thyroplasty type I in the treatment of unilateral vocal paralysis. Laryngoscope. 1996; 106:573-577. [PubMed: 8628083] 


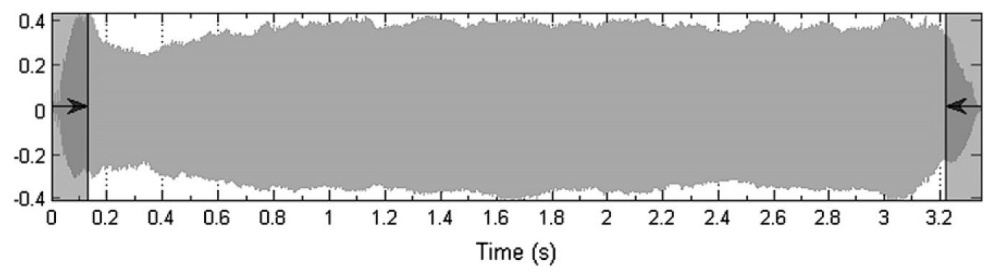

FIGURE 1.

Illustration of systematic trimming used to select useful parts of the sustained phonations for subsequent analysis by objective measures. The dark-shaded areas indicate the 0.12 -second portion removed at the start and end of the phonation, with exactly equal lengths in seconds from the start and end. This removal was applied uniformly across all phonations. 

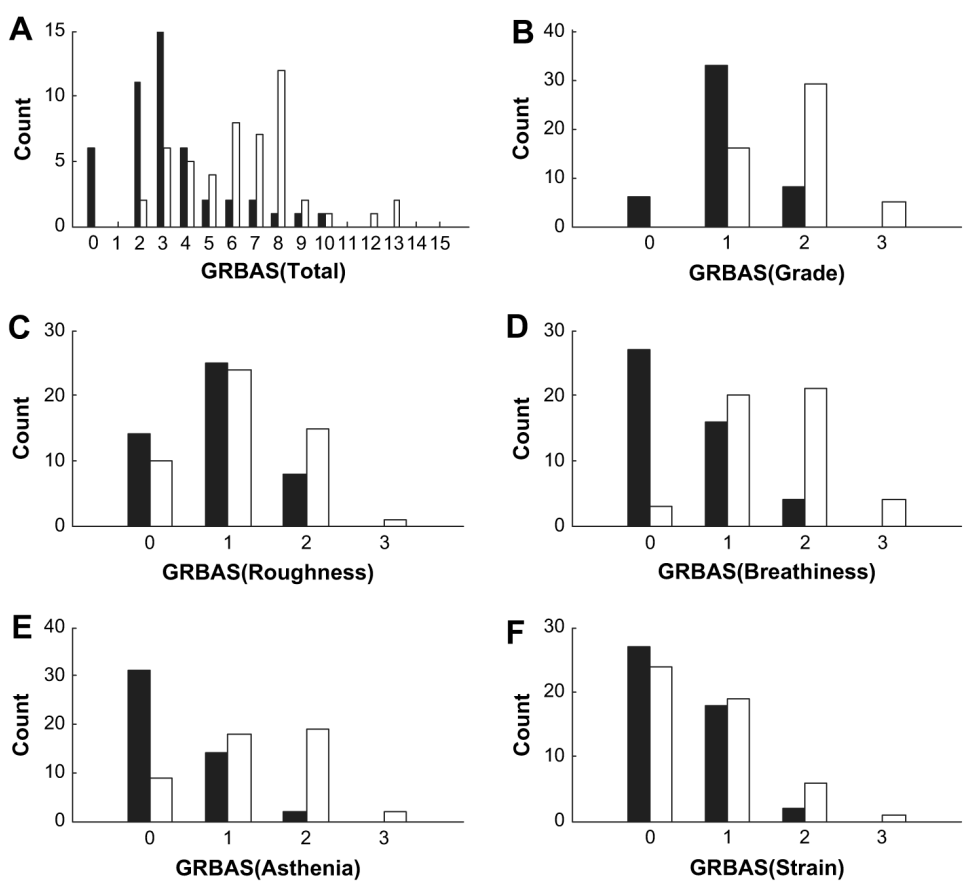

FIGURE 2.

Histograms of subjective GRBAS ratings for the UVFP phonations. Unfilled white bars are preoperative scores and filled black bars are postoperative scores. The horizontal axis shows the GRBAS score in each subcategory and the vertical axis shows the number of instances of that score. 

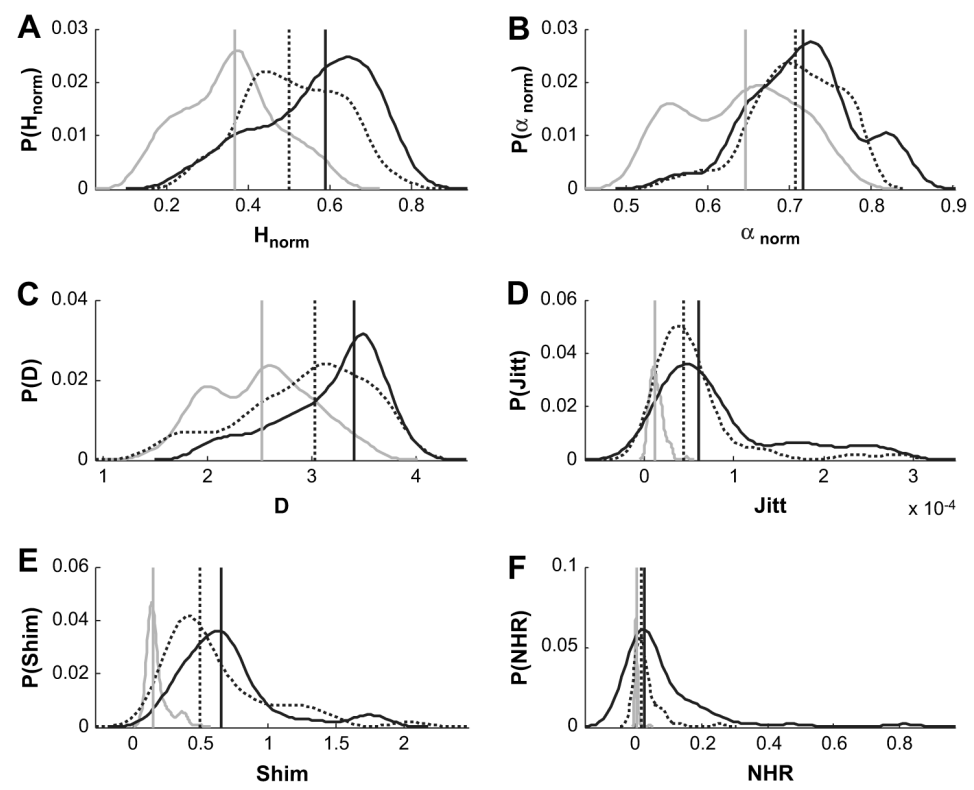

FIGURE 3.

Estimated probability of all measures: $(\mathbf{A})$ recurrence probability density, $(\mathbf{B})$ normalized detrended fluctuation, (C) correlation dimension, (D) absolute jitter (s), (E) shimmer (dB), (F) noise-to-harmonic ratio (NHR). See text for full algorithm descriptions. The probabilities for the three separate groups with normal voices (solid gray lines), preoperative UVFP (solid black lines), and postoperative UVFP (dashed black lines) are estimated using the kernel density method with Gaussian kernel. The vertical axes show the probabilities. The vertical lines are the median values of the measures for each group. 


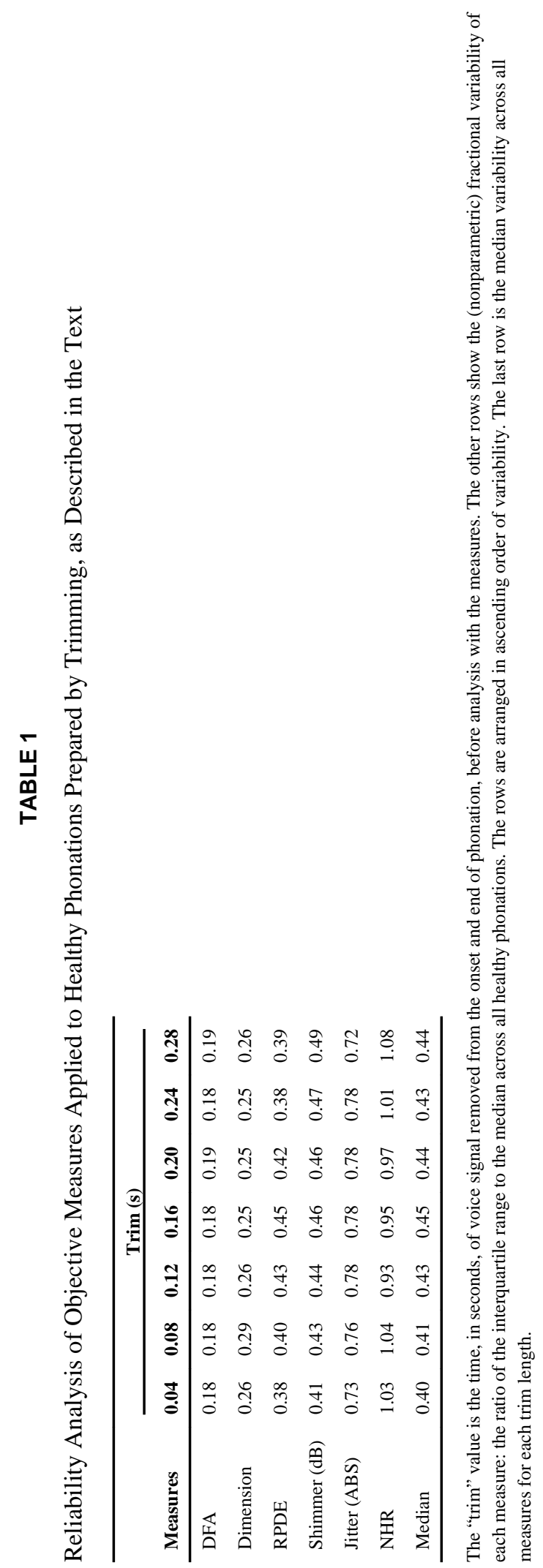




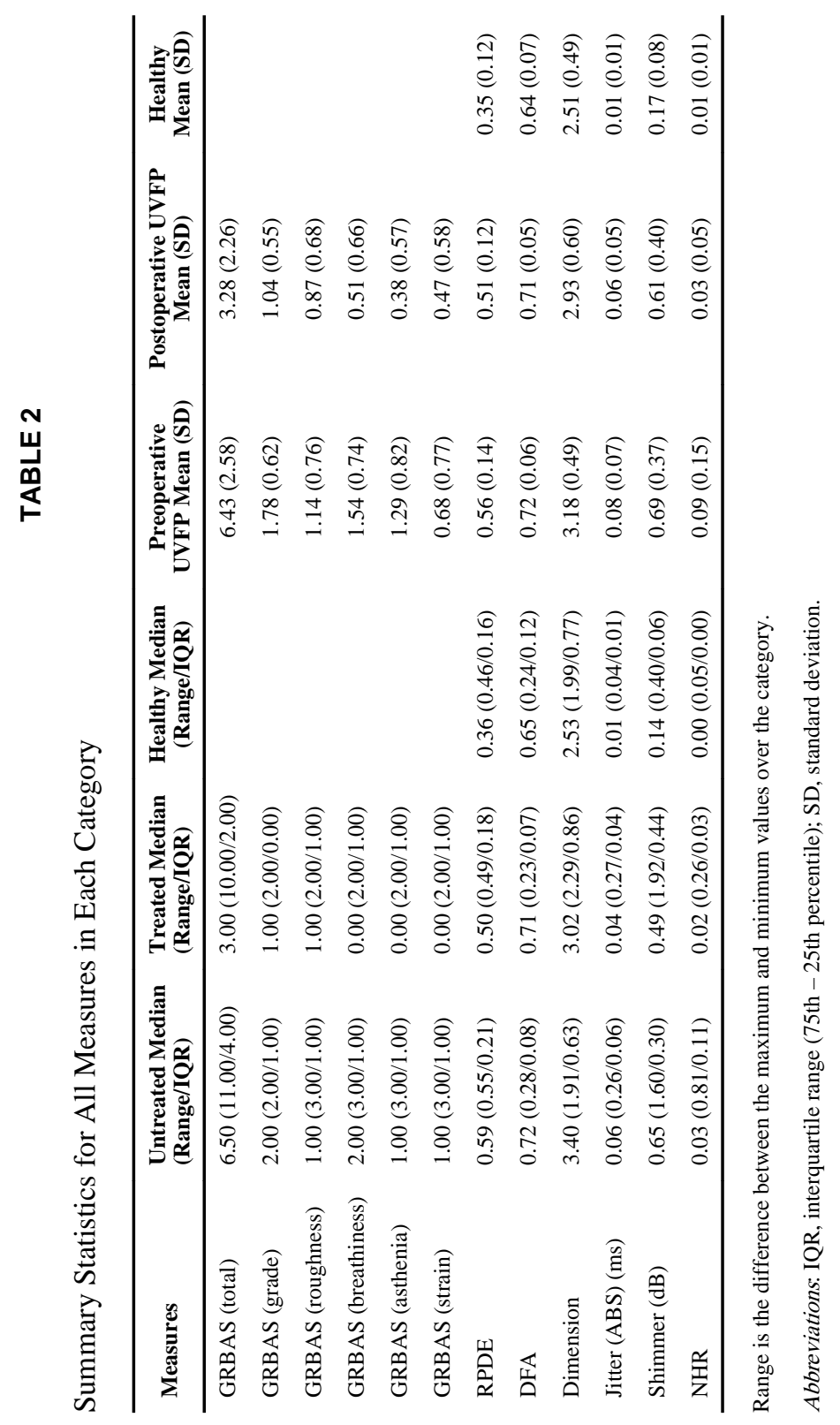

J Voice. Author manuscript; available in PMC 2011 August 23. 


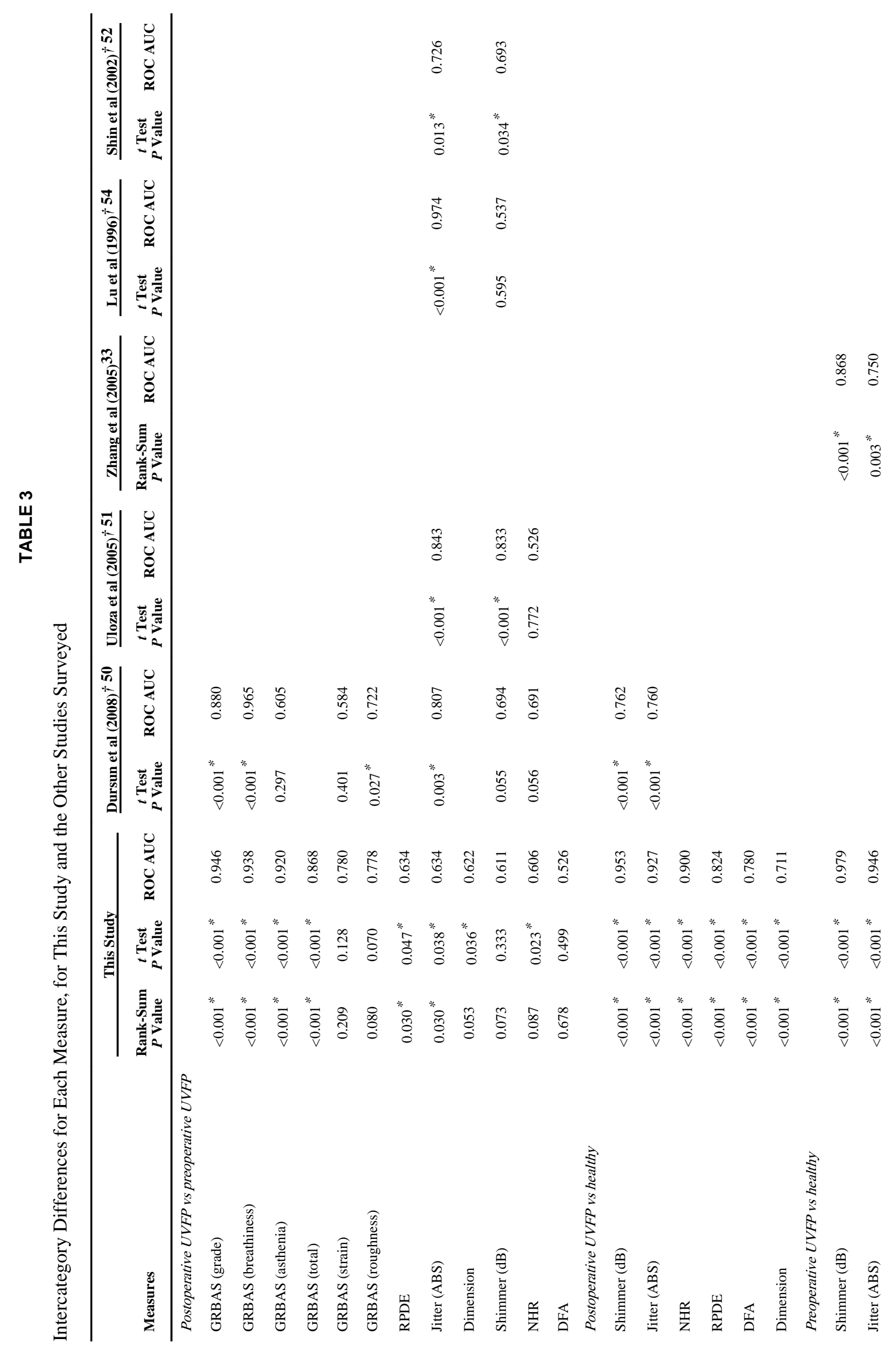




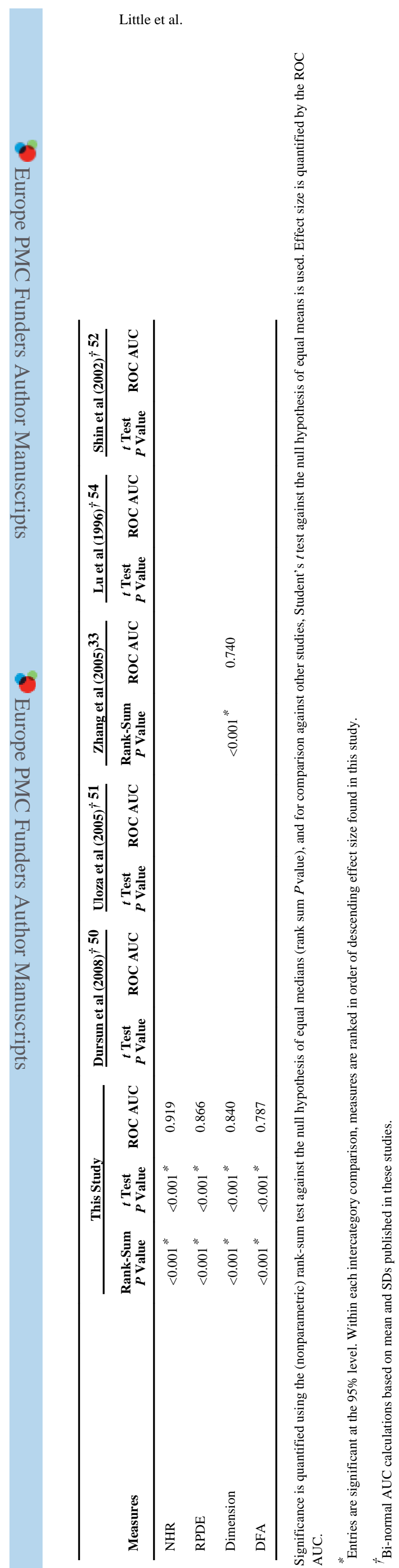

Page 20 